\title{
Paediatric crossword puzzle 28
}

\section{Manouri P Senanayake ${ }^{1}$}

Sri Lanka Journal of Child Health, 2015; 44(3): 178

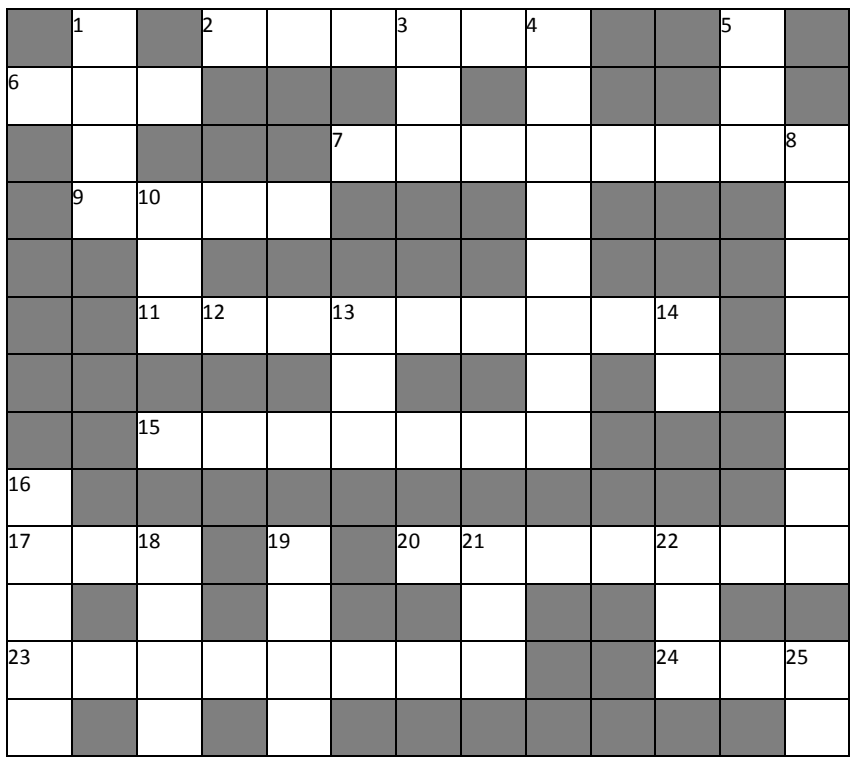

\section{ACROSS}

2. Pattern of depigmentation in Hypomelanosis of Ito.

6. Is used for feeding liquids rather than solids. 7. Antibiotic-steroid drops are clinically to oral antibiotics for treating otorrhoea in children with tympanostomy tubes.

9. Term synonymous with SIDS.

11. Element best avoided in antacids prescribed for infants.

15. Sleep at an early age is associated with sleepwalking.

17. Unipolar limb lead in ECG with positive electrode on right arm

20. Nifedipine decreases entry across cell membranes.

23. "Mucosal protective" compound used to treat gastro-oesophageal reflux disease.

24. Muscle fibres extensively replaced by adipose tissue ( abbrv)

\section{DOWN}

1. Extent of maxillary space in deciduous dentition of breast fed babies.

3. A tool used in USA to measure care doctors provide (abbrv)

4. Most commonly used medicine for interstitial lung disease in childhood.

5. World Immunization Week is an initiative of..............

8. A Contagious cause of localized alopecia. 10. Extensive research on possible link between MMR and Autism was carried out recently in (abbrv)

12. Munchausen Syndrome by proxy consists of

13. Vaccination falsely incriminated as causative of Autism.

14. Constituent of antacids causing a laxative effect (abbrv).

16. Stimulation that helps correct supraventricular tachycardia.

18. 'Out-of-control' behaviour in Asperger syndrome is called "blind- ....."

19. Recommended for childhood diarrhoea

21. Enzyme inhibitor used widely to treat heart failure in children.

22. Gastrointestinal disease that has increased with improved personal hygiene (abbrv).

25. An academic title dating back over a millennium, based on Latin for teacher 